DRAFT VERSION NOVEMBER 11, 2018

Preprint typeset using $\mathrm{LT}_{\mathrm{E}} \mathrm{X}$ style emulateapj v. 08/22/09

\title{
CYCLIC MAGNETIC ACTIVITY DUE TO TURBULENT CONVECTION IN SPHERICAL WEDGE GEOMETRY
}

\author{
Petri J. KÄPyl $\ddot{A}^{1}$, MAARIT J. MANTERE ${ }^{1}$ And AXEL BrandenburG ${ }^{2,3}$ \\ ${ }^{1}$ Physics Department, Gustaf Hällströmin katu 2a, PO Box 64, FI-00014 University of Helsinki, Finland \\ ${ }^{2}$ NORDITA, Royal Institute of Technology and Stockholm University, Roslagstullsbacken 23, SE-10691 Stockholm, Sweden \\ ${ }^{3}$ Department of Astronomy, AlbaNova University Center, Stockholm University, SE 10691 Stockholm, Sweden \\ Draft version November 11, 2018
}

\begin{abstract}
We report on simulations of turbulent, rotating, stratified, magnetohydrodynamic convection in spherical wedge geometry. An initially small-scale, random, weak-amplitude magnetic field is amplified by several orders of magnitude in the course of the simulation to form oscillatory large-scale fields in the saturated state of the dynamo. The differential rotation is solar-like (fast equator), but neither coherent meridional poleward circulation nor near-surface shear layer develop in these runs. In addition to a poleward branch of magnetic activity beyond 50 degrees latitude, we find for the first time a pronounced equatorward branch at around 20 degrees latitude, reminiscent of the solar cycle.
\end{abstract}

Subject headings: Magnetohydrodynamics - convection - turbulence

\section{INTRODUCTION}

The solar magnetic field exhibits a quasi-periodic cycle with a period of approximately 22 years. This cycle is manifested by the appearance of sunspots in low latitude activity belts that migrate towards the equator as the sunspot cycle progresses. Reproducing this behavior remains a major challenge to theoreticians. Mean-field models, where small-scale turbulent effects are parameterized (e.g. Krause \& Rädler 1980), have reproduced many aspects of the solar cycle, but with broadly varying assumptions for the various parameterizations (see, e.g. Dikpati \& Charbonneau 1999; Ossendrijver 2003; Käpylä et al. 2006; Kitchatinov \& Olemskoy 2012).

Another, computationally much more demanding, but physically more consistent route is to solve the equations of magnetohydrodynamics directly without resorting to ill-defined parameterizations for the small scales. In practise, however, realistic Reynolds and Rayleigh numbers, describing the effects of molecular diffusion with respect to advection, are not accessible to simulations (e.g. Chan \& Sofia 1986; Miesch \& Toomre 2009; Käpylä 2011). The usual approach is to enhance the diffusion coefficients to levels that are computationally feasible while striving to maximize the resolution.

Early spherical shell simulations were able to produce a solar-like rotation profile, i.e. one with "equatorward acceleration," and oscillatory large-scale dynamos (Gilman 1983; Glatzmaier 1985). However, the direction of propagation of the dynamo wave was towards the poles, in contradiction to the Sun. This can be qualitatively explained by a Parker dynamo wave with positive radial shear near the equator in conjunction with negative kinetic helicity density, or a positive $\alpha$-effect, in the northern hemisphere (Parker 1955). More sophisticated simulations with solar rotation rate and luminosity have failed to produce strong large-scale magnetic fields (Brun et al. 2004) or clear cyclic behavior (Miesch et al. 2011). These runs omitted a stable layer below the convection zone. When such a layer is added, nonoscillatory large-scale fields are found also for solar parameters (Browning et al. 2006). Later, oscillatory solutions have been obtained from similar simulations with subgrid-scale modeling (Ghizaru et al. 2010; Racine et al. 2011). These are

Electronic address: petri.kapyla@ helsinki.fi (Revision: 1.139) the most solar-like solutions so far, but also in them the activity is at too high latitudes and the activity belts do not propagate towards the equator. When the rotation rate is increased from the solar value in runs without an overshoot layer, first stable wreaths of strong large-scale fields appear (Brown et al. 2010), and at even more rapid rotation, poleward migrating activity is found (Käpylä et al. 2010; Brown et al. 2011).

We report here results from simulations of turbulent convection in spherical wedge geometry with solar-like equatorward acceleration that exhibit, for the first time, equatorward migrating magnetic activity near the equator and a polar branch at high latitudes. The numerical model is the same as that in Käpylä et al. (2011a) but here we add magnetic fields.

\section{THE MODEL}

We model a segment of a star, i.e. a "wedge", in spherical polar coordinates, where $(r, \theta, \phi)$ denote radius, colatitude, and longitude. The radial, latitudinal, and longitudinal extents of the wedge are $0.7 R \leq r \leq R, \theta_{0} \leq \theta \leq \pi-\theta_{0}$, and $0 \leq \phi \leq \phi_{0}$, respectively, where $R$ is the radius of the star. Here we take $\theta_{0}=\pi / 8$ and $\phi_{0}=\pi / 2$.

We solve the compressible hydromagnetics equations,

$$
\begin{gathered}
\frac{\partial \boldsymbol{A}}{\partial t}=\boldsymbol{u} \times \boldsymbol{B}-\eta \mu_{0} \boldsymbol{J}, \\
\frac{D \ln \rho}{D t}=-\boldsymbol{\nabla} \cdot \boldsymbol{u}, \\
\frac{D \boldsymbol{u}}{D t}=\boldsymbol{g}-2 \boldsymbol{\Omega} \times \boldsymbol{u}+\frac{1}{\rho}(\boldsymbol{J} \times \boldsymbol{B}-\boldsymbol{\nabla} p+\nabla \cdot 2 \nu \rho \mathbf{S}), \\
T \frac{D s}{D t}=\frac{1}{\rho}\left[\boldsymbol{\nabla} \cdot\left(K \boldsymbol{\nabla} T+\chi_{\mathrm{t}} \rho T \boldsymbol{\nabla} s\right)+2 \nu \mathbf{S}^{2}\right],
\end{gathered}
$$

where $\boldsymbol{A}$ is the magnetic vector potential, $\boldsymbol{u}$ is the velocity, $\boldsymbol{B}=\boldsymbol{\nabla} \times \boldsymbol{A}$ is the magnetic field, $\boldsymbol{J}=\mu_{0}^{-1} \nabla \times \boldsymbol{B}$ is the current density, $\eta$ is the magnetic diffusivity, $\mu_{0}$ is the vacuum permeability, $D / D t=\partial / \partial t+\boldsymbol{u} \cdot \boldsymbol{\nabla}$ is the advective time derivative, $\nu$ is the kinematic viscosity, $K$ is the radiative heat conductivity, $\chi_{\mathrm{t}}$ is the unresolved turbulent heat conductivity. $\rho$ is the density, $s$ is the specific entropy, $T$ is the temperature, and $p$ is the pressure. The fluid obeys the ideal gas law 
with $p=(\gamma-1) \rho e$, where $\gamma=c_{\mathrm{P}} / c_{\mathrm{V}}=5 / 3$ is the ratio of specific heats at constant pressure and volume, respectively, and $e=c_{\mathrm{V}} T$ is the internal energy. The gravitational acceleration is $\boldsymbol{g}=-G M \hat{\boldsymbol{r}} / r^{2}$, where $G$ is the gravitational constant, $M$ is the mass of the star, and $\hat{\boldsymbol{r}}$ is the unit vector in the radial direction. We omit the centrifugal force (cf. Käpylä et al. 2011b). The rate of strain tensor $\mathbf{S}$ is given by $\mathrm{S}_{i j}=\frac{1}{2}\left(u_{i ; j}+u_{j ; i}\right)-\frac{1}{3} \delta_{i j} \boldsymbol{\nabla} \cdot \boldsymbol{u}$, where the semicolons denote covariant differentiation (Mitra et al. 2009).

\subsection{Initial and boundary conditions}

The initial state is isentropic and the hydrostatic temperature gradient is $\partial T / \partial r=-g /\left[c_{\mathrm{V}}(\gamma-1)(m+1)\right]$, where $m=1.5$ is the polytropic index. We fix the value of $\partial T / \partial r$ on the lower boundary. The density profile follows from hydrostatic equilibrium. The heat conduction profile is chosen so that radiative diffusion is responsible for supplying the energy flux in the system, with $K$ decreasing more than two orders of magnitude from bottom to top (Käpylä et al. 2011a). A weak random small-scale seed magnetic field is taken as initial condition (see below).

The radial and latitudinal boundaries are taken to be impenetrable and stress free; see Equations (14) and (15) of Käpylä et al. (2011b). For the magnetic field we assume perfect conductors at the lower radial and latitudinal boundaries, and radial field at the outer radial boundary; see Equations (15)-(17) of Käpylä et al. (2010). On the latitudinal boundaries we assume that the thermodynamic quantities have zero first derivatives, thus suppressing heat fluxes through the boundaries.

On the upper boundary we apply a black body condition

$$
\sigma T^{4}=-K \frac{\partial T}{\partial r}-\chi_{\mathrm{t}} \rho T \frac{\partial s}{\partial r},
$$

where $\sigma$ is the Stefan-Boltzmann constant. We use a modified value for $\sigma$ that takes into account that our Reynolds and Rayleigh numbers are much smaller than in reality, so $K$ and therefore the flux are much larger than in the Sun.

\subsection{Dimensionless parameters}

We obtain non-dimensional quantities by choosing $R=$ $G M=\rho_{0}=c_{\mathrm{P}}=\mu_{0}=1$, where $\rho_{0}$ is the initial density at $0.7 R$. Our simulations are defined by the energy flux imposed at the bottom boundary, $F_{\mathrm{b}}=-\left.(K \partial T / \partial r)\right|_{r=0.7 R}$, the temperature at the top boundary, $T_{1}=T(r=R)$, as well as the values of $\Omega_{0}, \nu, \eta$, and $\chi_{\mathrm{tm}}=\chi_{\mathrm{t}}\left(r_{\mathrm{m}}=0.85 R\right)$. The corresponding nondimensional input parameters are the luminosity parameter $\mathcal{L}=L_{0} /\left[\rho_{0}(G M)^{3 / 2} R^{1 / 2}\right]$, the normalized pressure scale height at the surface, $\xi=\left[(\gamma-1) c_{\mathrm{V}} T_{1}\right] R / G M$, the Taylor number Ta $=\left(2 \Omega R^{2} / \nu\right)^{2}$, the Prandtl number $\operatorname{Pr}=\nu / \chi_{\mathrm{tm}}$, the magnetic Prandtl number $\operatorname{Pm}=\nu / \eta$, and the non-dimensional viscosity $\tilde{\nu}=\nu / \sqrt{G M R}$. Other useful diagnostic parameters are the Reynolds number $\mathrm{Re}=$ $u_{\mathrm{rms}} / \nu k_{\mathrm{f}}$ and the Coriolis number Co $=2 \Omega_{0} / u_{\mathrm{rms}} k_{\mathrm{f}}$, where $u_{\mathrm{rms}}=\sqrt{(3 / 2)\left\langle u_{r}^{2}+u_{\theta}^{2}\right\rangle}$ is the rms velocity. Note that for $u_{\mathrm{rms}}$ we omit the contribution from the azimuthal velocity, because its value is dominated by effects from the differential rotation (Käpylä et al. 2011b). The Taylor number can also be written as $\mathrm{Ta}=\mathrm{Co}^{2} \operatorname{Re}^{2}\left(k_{\mathrm{f}} R\right)^{4}$, with $k_{\mathrm{f}} R \approx 21$. Due to the fact that the initial stratification is isentropic, we quote the (semi-) turbulent Rayleigh number $\mathrm{Ra}_{\mathrm{t}}$ from the thermally re- laxed state of the run,

$$
\mathrm{Ra}_{\mathrm{t}}=\frac{G M(\Delta r)^{4}}{\nu \chi_{\mathrm{tm}} R^{2}}\left(-\frac{1}{c_{\mathrm{P}}} \frac{\mathrm{d} s}{\mathrm{~d} r}\right)_{r_{\mathrm{m}}},
$$

where $k_{\mathrm{f}}=2 \pi / \Delta r$ is an estimate of the wavenumber of the largest eddies, and $\Delta r=0.3 R$ is the thickness of the layer. The magnetic field is expressed in equipartition field strengths, $B_{\text {eq }}(r)=\left\langle\mu_{0} \rho \boldsymbol{u}^{2}\right\rangle_{\theta \phi}^{1 / 2}$, where the subscripts indicate averaging over $\theta$ and $\phi$ with azimuthally averaged mean flows subtracted.

The simulations were performed with the PENCIL CODE ${ }^{1}$, which is a high-order finite difference method for solving the compressible equations of magnetohydrodynamics.

\section{RESULTS}

Our primary simulation (Run B4m) is continued from a thermally relaxed snapshot of a hydrodynamic Run B4 of Käpylä et al. (2011a) with $\mathcal{L}=3.8 \cdot 10^{-5}, \xi=0.02$, $\mathrm{Ta} \approx 1.4 \times 10^{10}, \tilde{\nu}=2.9 \times 10^{-5}$, and $\operatorname{Pr}=2.5$, resulting in $\mathrm{Re}=36, \mathrm{Co}=7.6$ and $\mathrm{Ra}_{\mathrm{t}} \approx 3 \cdot 10^{6}$. The discussion of the results refers to this run unless stated otherwise. We also consider two other runs with $\mathrm{Co}=4.7$ and $\mathrm{Re}=39$ (Run B3m), as well as $\mathrm{Co}=14.8$ and $\mathrm{Re}=31$ (Run B5m). The former is continued from Run B3 of Käpylä et al. (2011a) whereas the latter is run from the initial conditions stated above. Our seed magnetic field has an amplitude of $\approx 10^{-4} B_{\text {eq }}$. As a starting point, we use $\mathrm{Pm}=1$ and a resolution of $128 \times 256 \times 128$ mesh points, but also study the magnetic Prandtl number dependence by continuing Run B4m with values $\mathrm{Pm}=(0.25,0.5)$. In Run $\mathrm{B} 4 \mathrm{~m}$ the magnetic field grows exponentially over roughly 1500 convective turnover times before reaching the saturated stage in which the total rms magnetic field is $B_{\mathrm{rms}}=0.72 B_{\text {eq }}$.

The convection pattern near the surface shows smaller scales at high latitudes and larger elongated structures or 'banana cells' near the equator. Figure 1 shows the rotation profile in the saturated regime of the dynamo from Run B4m. The equator rotates faster than the high latitudes and significant radial differential rotation is present only near the equator. In the lower part of the convection zone, $\partial \bar{\Omega} / \partial r$ is negative at low latitudes (just outside the inner tangent cylinder) and positive at high latitudes. The meridional circulation shows several small cells outside the tangent cylinder on both hemispheres. The latitudinal differential rotation, measured by $\Delta_{\Omega} \equiv\left(\Omega_{\theta=\theta_{0}}-\Omega_{\mathrm{eq}}\right) / \Omega_{\mathrm{eq}}$, where $\Omega_{\mathrm{eq}}=\Omega(\theta=\pi / 2)$, decreases from 0.08 in the kinematic regime to 0.07 in the saturated state. The rotation profiles in Runs B3m and B5m are qualitatively similar.

We define mean quantities as averages over longitude and denote them by an overbar. In Run B4m the relative kinetic helicity density $h_{\mathrm{rel}}=\overline{\boldsymbol{u} \cdot \boldsymbol{\omega}} / u_{\mathrm{rms}} \omega_{\mathrm{rms}}$, with $\boldsymbol{\omega}=\boldsymbol{\nabla} \times \boldsymbol{u}$, is negative (positive) in the northern (southern) hemisphere; see Figure 11 No pronounced sign reversal with depth is seen. The maximum value of $h_{\text {rel }}$ is around 0.3 , which allows us to determine the dynamo number describing the strength of the $\alpha$ effect as $C_{\alpha}=\alpha / \eta_{\mathrm{t} 0} k_{1} \approx h_{\mathrm{rel}} k_{\mathrm{f}}^{(\omega)} / k_{1} \approx 2.7$, where $k_{\mathrm{f}}^{(\omega)}=\omega_{\mathrm{rms}} / u_{\mathrm{rms}}$ is the approximate wavenumber of the energy-carrying eddies, $k_{1}=\pi / \Delta r$ is the lowest radial wavenumber in the domain, while $\alpha \approx h_{\text {rel }} u_{\mathrm{rms}} / 3$ and $\eta_{\mathrm{t} 0}=u_{\mathrm{rms}} / 3 k_{\mathrm{f}}^{(\omega)}$ are estimates for $\alpha$ effect and turbulent

\footnotetext{
${ }^{1} \mathrm{http} / / /$ code.google.com/p/pencil-code/
} 

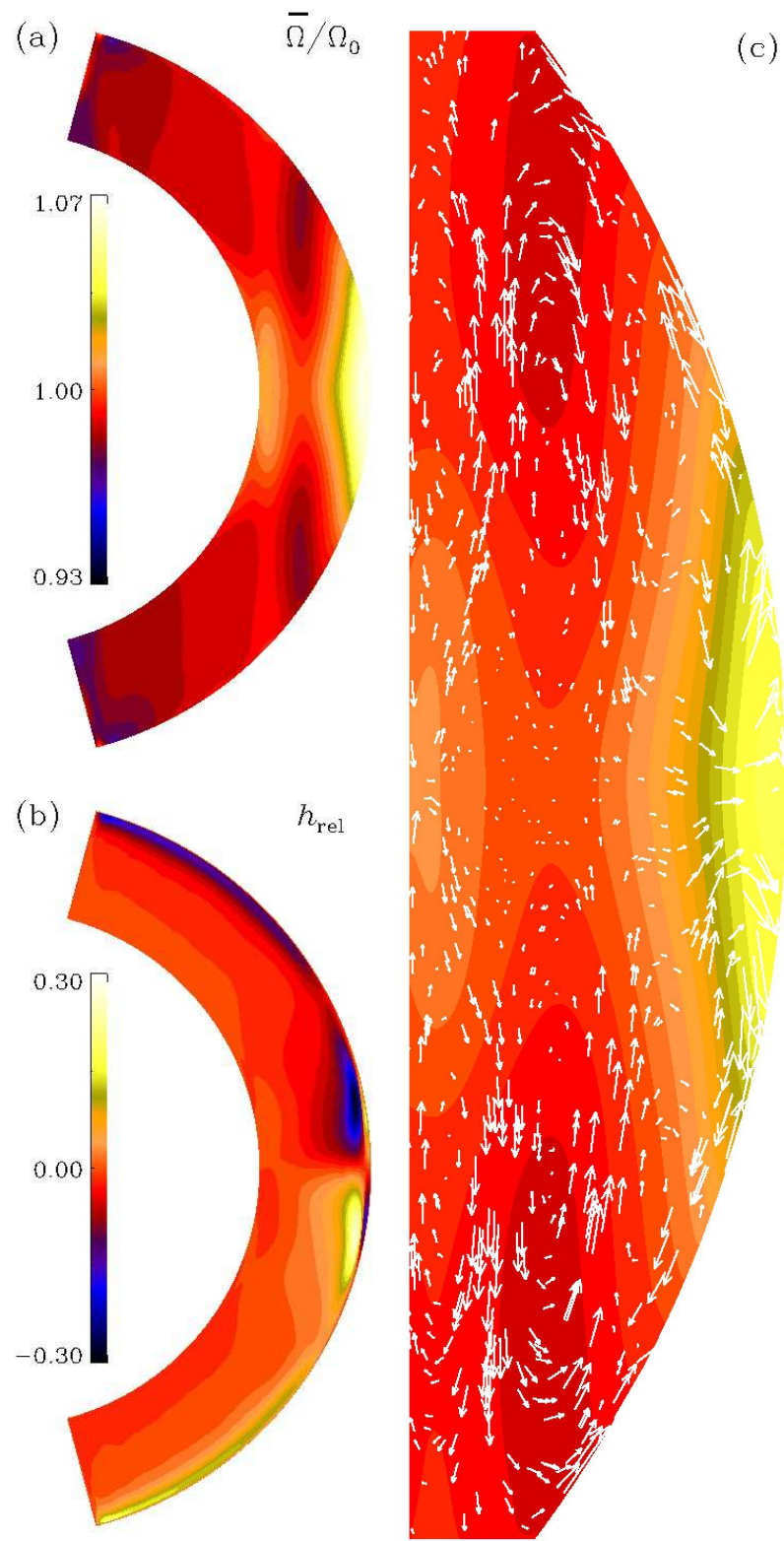

FIG. 1.- (a) Normalized time-averaged mean rotation profile $\bar{\Omega} / \Omega_{0}=$ $\bar{U}_{\phi} /\left(\Omega_{0} r \sin \theta\right)+1$. (b) Relative kinetic helicity density $h_{\text {rel }}$. (c) Rotation profile (color contours) and meridional circulation $\overline{\boldsymbol{U}}_{m}=\left(\bar{U}_{r}, \bar{U}_{\theta}, 0\right)$ (arrows) near the equator. From Run B4m.

magnetic diffusivity. (We note that $\chi_{\mathrm{t}} / \eta_{\mathrm{t} 0}$ varies between 1.9 near the surface and 0.15 within the convection zone.) The relevant dynamo number characterizing the radial differential rotation is $C_{\Omega}=\Delta \Omega / \eta_{\mathrm{t} 0} k_{1}^{2} \approx 55$, where we have used $\Delta \Omega / \Omega_{0}=0.06$ for the normalized radial shear (not to be confused with $\Delta_{\Omega}$ defined above). The ratio $C_{\Omega} / C_{\alpha}$ is well over 10. Following Roberts \& Stix (1972), this suggests that we are in what is known as the $\alpha \Omega$ regime where shear is strong enough to favor cyclic behavior.

Time series of the averaged longitudinal component of the magnetic field are shown in Figure 2 for different values of $\mathrm{Co}=(4.7,7.6$, and 14.8). For $\mathrm{Co}=7.6$, two activity belts are visible; one propagating poleward at high latitudes and another propagating equatorward between 10 and 30 degrees latitude. The equatorward branch at mid-latitudes is visible also for $\mathrm{Co}=4.7$ but there the cycle is irregular and the mag-
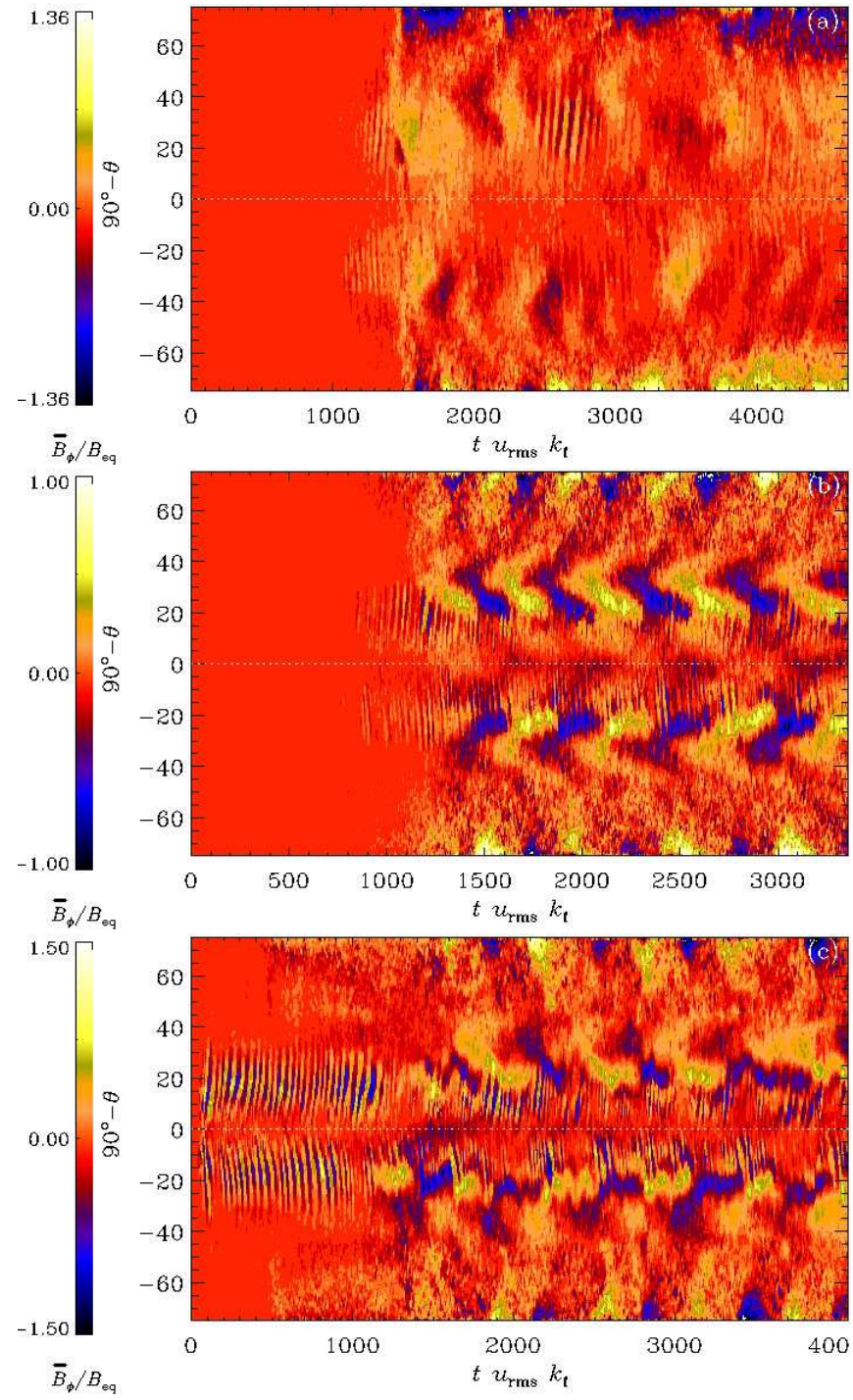

FIG. 2. $-\bar{B}_{\phi}$ near the surface of the star at $r=0.98 R$ as a function of latitude $90^{\circ}-\theta$ for $\mathrm{Co}=4.7$ (top, Run B3m), 7.6 (middle, B4m), and 14.8 (bottom, B5m). The white dotted line denotes the equator $90^{\circ}-\theta=0$.

netic field at low latitudes weaker. The dynamo mode appears to change to a non-oscillatory one after around $3800 t u_{\mathrm{rms}} k_{\mathrm{f}}$. In the most rapidly rotating case with $\mathrm{Co}=14.8$ the cyclicity and equatorward migration of the field are clearly present but fluctuations from one cycle to the next are again larger than in Run B4m. In all of the runs a poleward branch with a shorter period is visible near the surface at low latitudes which looks similar to the solution obtained in the nonlinear stage in Käpylä et al. (2010). This mode can be occasionally distinguished in the saturated stage, in particular in Run B5m, but it remains subdominant to the mode with longer period exhibiting equatorward migration.

The magnetic field is strongest at $r / R \approx 0.85$ and seems to propagate from there to top and bottom of the convection zone; see Figure 3 (a) which shows $\bar{B}_{\phi}$ as a function of $r$ and $t$ being regenerated in the bulk of the convection zone during each cycle. As the field approaches the surface, it propagates equatorward at low latitudes; see Figure 3 (b). This mode becomes apparent in the nonlinear phase whereas in the kinematic stage the solution in the bulk of the convection zone does not oscillate; see Figure 3 (c) for $t u_{\mathrm{rms}} k_{\mathrm{f}}<1000$. Since 

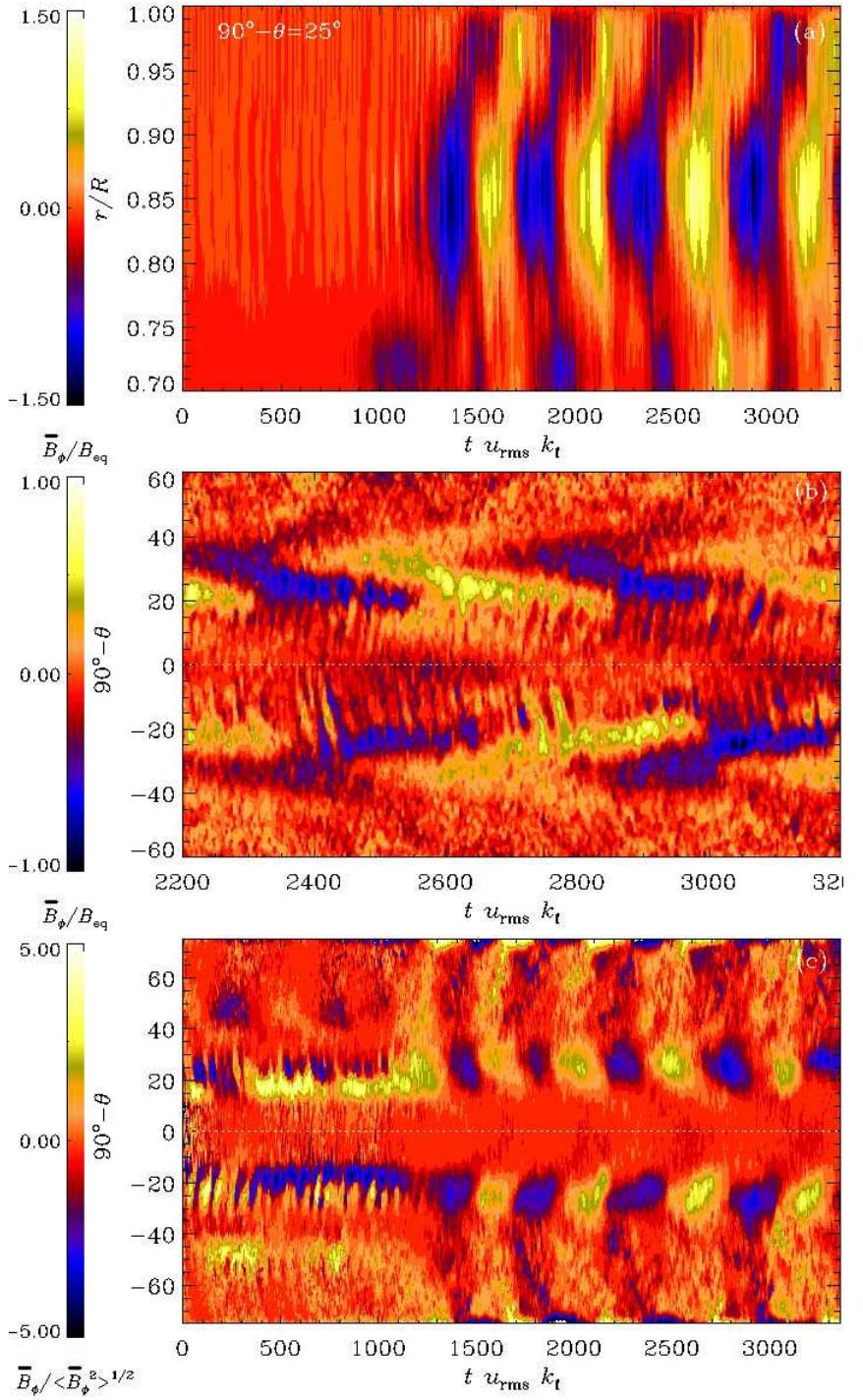

FIG. 3.- (a) $\bar{B}_{\phi}(r, t)$ in units of the local equipartition field strength at $25^{\circ}$ latitude for Run B4m shown in Figure 2 b). (b) Blow-up of Figure 2 b) showing the region $-60^{\circ}<90^{\circ}-\theta<60^{\circ}$ and $2200<t u_{\mathrm{rms}} k_{\mathrm{f}}<$ 3200 at $r=0.98 R$. (c) Like Figure 2 b), but at $r=0.85 R$ and $\bar{B}_{\phi}$ is normalized by its volume averaged rms-value at each time to make the early time evolution visible.

$\bar{B}_{\phi}$ is here normalized by the instantaneous average value, one sees the spatio-temporal structure, and that no reversals occur. Opposite transitions (from oscillatory to quasi-steady) have been observed in Cartesian simulations (Hubbard et al. 2011; Käpylä et al. 2012).

On theoretical grounds, we would expect $\left|\bar{B}_{\phi} / \bar{B}_{r}\right|$ to be of the order of $\left|C_{\Omega} / C_{\alpha}\right|^{1 / 2} \approx 4.5$, but the actual ratio is only around unity; see Figure 4. We cannot therefore be certain that the dynamo is really in the $\alpha \Omega$ regime, as discussed above. Interestingly, $\bar{B}_{r}$ shows a greater amplitude at high latitudes while $\bar{B}_{\phi}$ is stronger at lower latitudes. Furthermore, $\bar{B}_{r}$ at high latitudes changes sign approximately when $\bar{B}_{\phi}$ in the low-latitude activity belt changes sign.

Visualizations of the toroidal magnetic field from Run B4m near the surface (Figure 5) show a persistent activity belt near the equator which is changing polarity with a period of roughly $400 \tau$ where $\tau=\left(u_{\mathrm{rms}} k_{\mathrm{f}}\right)^{-1}$ is the convective turnover time. We find that decreasing $\mathrm{Rm}$ to $18(\mathrm{Pm}=0.5)$
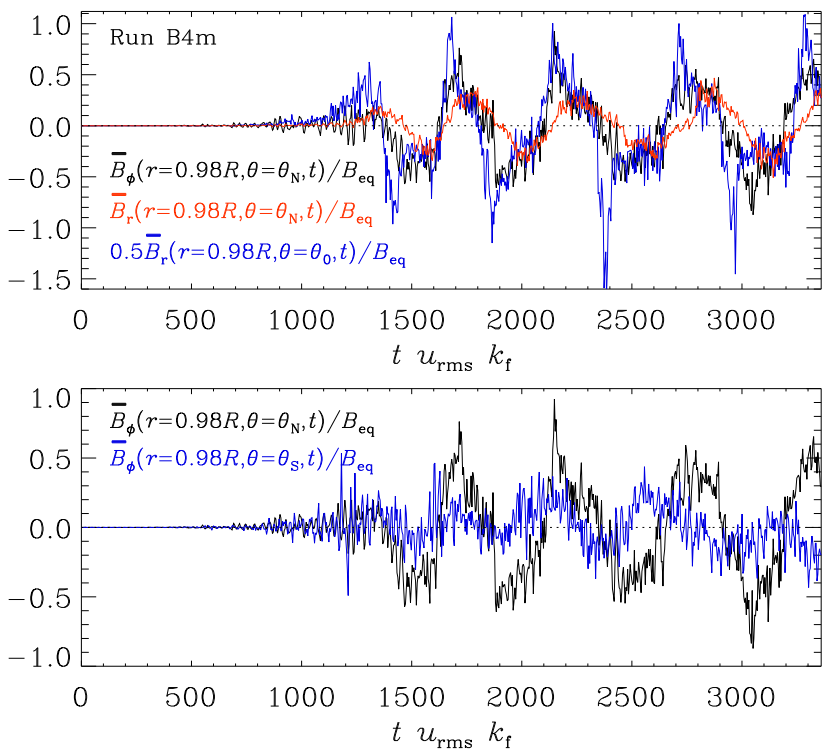

FIG. 4.- Top panel: $\bar{B}_{\phi}$ (black line) and $\bar{B}_{r}$ (red) at $90^{\circ}-\theta_{\mathrm{N}}=25^{\circ}$ latitude. The blue line shows $0.5 \bar{B}_{r}$ at $\theta_{0}$. Bottom panel: $\bar{B}_{\phi}$ from $\theta_{\mathrm{N}}$ and $\theta_{\mathrm{S}}$ corresponding to latitudes \pm 25 degrees, respectively.

shortens the cycle period by roughly 20 percent to $330 \tau$. At $\mathrm{Rm}=9(\mathrm{Pm}=0.25)$ the magnetic field decays, but the decay mode is oscillatory with a period of $270 \tau$. Similar increase of the cycle period with $\mathrm{Rm}$ has been observed in forced turbulence simulations (Käpylä \& Brandenburg 2009) and suggests that the current runs are not in a regime where the molecular diffusivities are unimportant. The same cyclic behavior is seen throughout the depth of the convection zone above $r=0.75 R$. Relating the turnover time of our highest $\mathrm{Rm}$ model to that of the deep layers of the solar convection zone, i.e. one month, leads to a magnetic cycle period of roughly 33 years. The cycle might well be shorter if the relevant depth is shallower. On the other hand, if we used $k_{\mathrm{f}}^{(\omega)}$ instead of $k_{\mathrm{f}}$, our cycle period would be $4-5$ times longer. It is also noteworthy that $\bar{B}_{\phi}$ has mixed parity about the equator, except around the time $t u_{\mathrm{rms}} k_{\mathrm{f}}=2500$ when the field is of odd parity; see Figure 4.

\section{CONCLUSIONS}

We have reported solar-like magnetic cycles from simulations of turbulent convection in spherical wedge geometry. The magnetic activity is concentrated in two belts, a high-latitude one propagating poleward, and a low-latitude one propagating equatorward. The strongest magnetic fields, however, occur in the high-latitude activity branch. Simulations with moderately slower and faster rotation show similar behavior. These results will be discussed in more detail in forthcoming publications. Relating the convective turnover time in the simulation to that of the Sun we obtain a cycle period of 33 years which is somewhat longer than that in the Sun and half that obtained by Ghizaru et al. (2010) from quite a different model exhibiting similar solutions, but without equatorward migration. One of the main differences to our earlier work (Käpylä et al. 2010) is that we have omitted a stably stratified overshoot layer beneath. This allowed us to cover almost an order of magnitude larger density contrast within the convectively unstable layer. Furthermore, convective energy transport now dominates over radiative diffusion and a black body boundary condition is used for the 

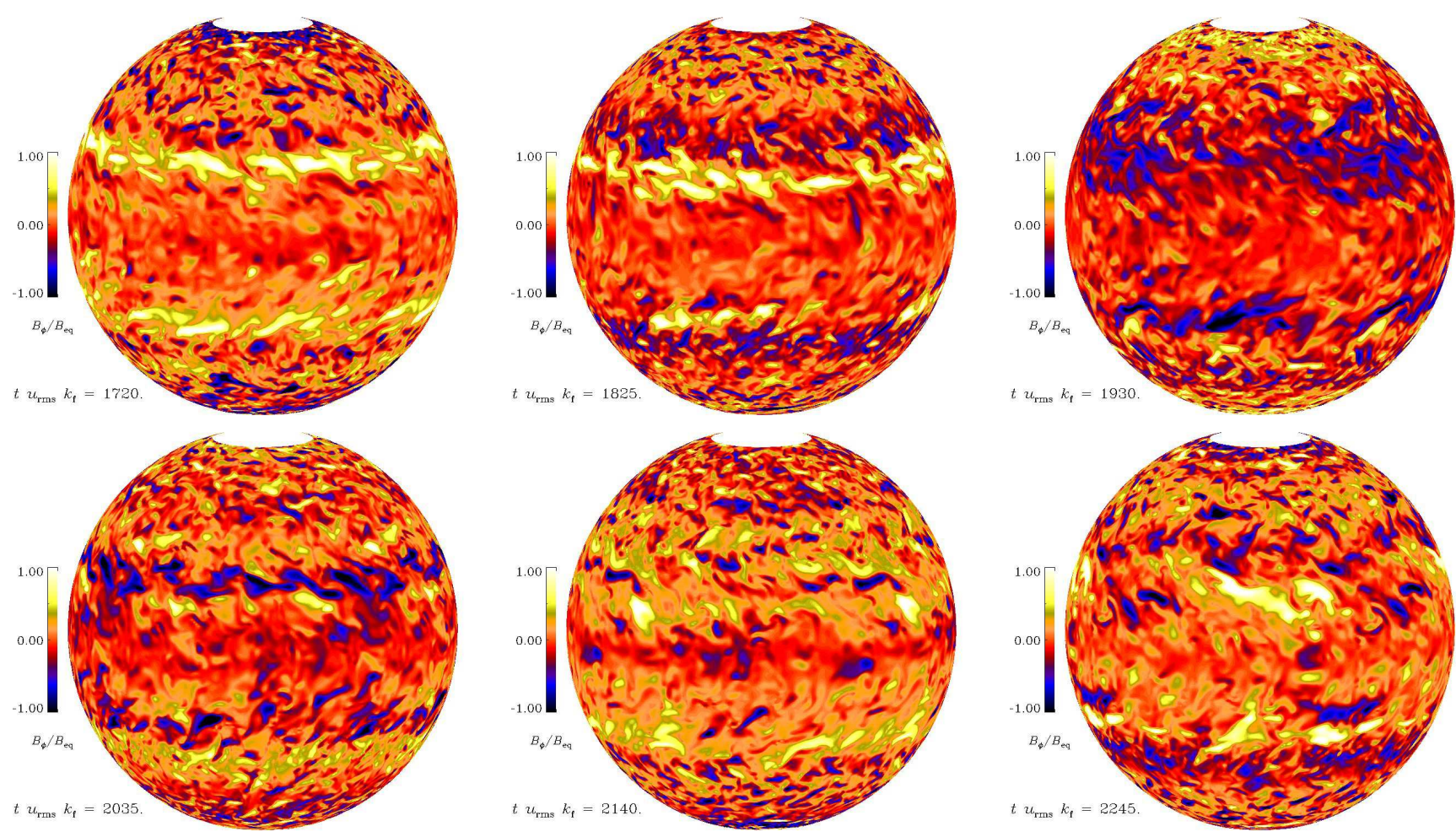

FIG. 5.- Snapshots of the toroidal magnetic field $B_{\phi}$ at $r=0.98 R$ from Run B4m at six different times separated by $\Delta t u_{\mathrm{rms}} k_{\mathrm{f}} \approx 105$.

temperature (cf. Käpylä et al. 2011a). However, compared with the Sun, our contours of differential rotation are still too cylindrical and also the banana-cell pattern of radial velocity might not be realistic. Both may be a consequence of having a large Taylor number; even the turbulent Taylor number, $\left(\nu / \nu_{\mathrm{t}}\right)^{2} \mathrm{Ta}=9 \mathrm{Ta} / \operatorname{Re}^{2} \approx 10^{8}$, is rather large. Here, $\nu_{\mathrm{t}} \approx \eta_{\mathrm{t} 0}$ has been used as an estimate of the turbulent viscosity. The magnetic activity in our model is distributed throughout the convection zone, in contrast to the widely accepted fluxtransport dynamo mechanism (Dikpati \& Charbonneau 1999) in which a one-cell anti-clockwise (north) meridional circulation is crucial. In our model, meridional flows are convergent toward low latitudes (see Figure 11) and may contribute to the resulting equatorward migration. On the other hand, the negative radial differential rotation in the near-surface shear layer (as anticipated by Brandenburg 2005), which is here absent, is not the explanation for the resulting equatorward migration. Clarifying this is an important goal for future work.

The authors acknowledge the anonymous referee for constructive comments on the manuscript. The simulations were performed using the supercomputers hosted by CSC - IT Center for Science Ltd. in Espoo, Finland, who are administered by the Finnish Ministry of Education. Financial support from the Academy of Finland grants No. 136189, 140970 (PJK) and 218159, 141017 (MJM), as well as the Swedish Research Council grant 621-2007-4064, and the European Research Council under the AstroDyn Research Project 227952 are acknowledged. The authors thank NORDITA for hospitality during their visits.

\section{REFERENCES}

Brandenburg, A. 2005, ApJ, 625, 539

Brown, B. P., Browning, M. K., Brun, A. S., Miesch, M. S., \& Toomre, J. 2010, ApJ, 711, 424

Brown, B. P., Miesch, M. S., Browning, M. K., Brun, A. S., \& Toomre, J. 2011, ApJ, 731, 69

Browning, M. K., Miesch, M. S., Brun, A. S., \& Toomre, J. 2006, ApJ, 648, L157

Brun, A. S., Miesch, M. S., \& Toomre, J. 2004, ApJ, 614, 1073

Chan, K. L., \& Sofia, S. 1986, ApJ, 307, 222

Dikpati, M., \& Charbonneau, P. 1999, ApJ, 518, 508

Ghizaru, M., Charbonneau, P., \& Smolarkiewicz, P. K. 2010, ApJ, 715, L133

Gilman, P. A. 1983, ApJS, 53, 243

Glatzmaier, G. A. 1985, ApJ, 291, 300

Hubbard, A., Rheinhardt, M., \& Brandenburg, A. 2011, A\&A, 535, A48

Käpylä, P. J. 2011, Astron. Nachr., 332, 43

Käpylä, P. J., \& Brandenburg, A. 2009, ApJ, 699, 1059

Käpylä, P. J., Korpi, M. J., Brandenburg, A., Mitra, D., \& Tavakol, R. 2010, Astron. Nachr., 331, 73

Käpylä, P. J., Korpi, M. J., \& Tuominen, I. 2006, Astron. Nachr., 327, 884

Käpylä, P. J., Mantere, M. J., \& Brandenburg, A. 2011a, Astron. Nachr., 332,883
—. 2012, Geophys. Astrophys. Fluid Dyn., in press, arXiv:1111.6894

Käpylä, P. J., Mantere, M. J., Guerrero, G., Brandenburg, A., \& Chatterjee, P. 2011b, A\&A, 531, A162

Kitchatinov, L. L., \& Olemskoy, S. V. 2012, Sol. Phys., 276, 3

Krause, F., \& Rädler, K.-H. 1980, Mean-field Magnetohydrodynamics and Dynamo Theory (Oxford: Pergamon Press)

Miesch, M. S., Brown, B. P., Browning, M. K., Brun, A. S., \& Toomre, J.

2011, in IAU Symp., Vol. 271, Astrophysical Dynamics: From Stars to

Galaxies, ed. N. H. Brummell, A. S. Brun, M. S. Miesch, \& Y. Ponty, 261

Miesch, M. S., \& Toomre, J. 2009, Ann. Rev. Fluid Mech., 41, 317

Mitra, D., Tavakol, R., Brandenburg, A., \& Moss, D. 2009, ApJ, 697, 923

Ossendrijver, M. 2003, A\&A Rev., 11, 287

Parker, E. N. 1955, ApJ, 122, 293

Racine, É., Charbonneau, P., Ghizaru, M., Bouchat, A., \& Smolarkiewicz,

P. K. 2011, ApJ, 735, 46

Roberts, P. H., \& Stix, M. 1972, A\&A, 18, 453 